\title{
Quantum read-out and fast initialization of nuclear spin qubits with electric currents
}

\author{
Noah Stemeroff and Rogério de Sousd* \\ Department of Physics and Astronomy, University of Victoria, Victoria, B.C., V8W 3P6, Canada
}

(Dated: November 21, 2018)

\begin{abstract}
Nuclear spin qubits have the longest coherence times in the solid state, but their quantum readout and initialization is a great challenge. We present a theory for the interaction of an electric current with the nuclear spins of donor impurities in semiconductors. The theory yields a sensitivity criterion for quantum detection of nuclear spin states using electrically detected magnetic resonance, as well as an all electrical method for fast nuclear spin qubit initialization.
\end{abstract}

PACS numbers: 03.65.Yz; 03.67.Lx; 76.70.-r.

Running a current over a localized electron spin provides an extremely sensitive method to detect electron spin resonance [1]. This technique, commonly referred to as "electrically detected magnetic resonance" (EDMR) is now experiencing a revival in the context of solid state quantum computing [2]. Several interesting recent results [3 12] point to the possibility of using EDMR for quantum read-out of individual donor nuclear spin states in silicon. The hope is that EDMR will be the "silicon alternative" to optically detected magnetic resonance, a method that can detect single nuclear spin states of defects in diamond [13]. Nuclear spins in silicon are exceptional quantum memory units, with coherence times $T_{2 n}$ over a few seconds $[14,15]$. While single spin detection of donor electrons in silicon was demonstrated recently [16], the corresponding read-out of individual nuclear spins remains a great open problem.

Nuclear spin read-out using EDMR [ $[6]$ is based on the interaction between donor electron $(\boldsymbol{S})$ and nuclear $(\boldsymbol{I})$ spin in an external magnetic field $B$ :

$$
\mathcal{H}_{e n}=\omega_{e} S_{z}-\omega_{n} I_{z}+A \boldsymbol{S} \cdot \boldsymbol{I},
$$

where $\omega_{e}=g_{e} \mu_{e} B / \hbar$ and $\omega_{n}=g_{n} \mu_{n} B / \hbar$ are the Zeeman frequencies for electron and nuclear spins, respectively. The hyperfine interaction $A$ couples the donor electron to its nuclear spin. From the approximation $\mathcal{H}_{e n} \approx\left(\omega_{e}+A I_{z}\right) S_{z}$, we see that the electron spin resonance frequency will depend on the nuclear spin state. The EDMR experiment consists in applying microwaves at a fixed frequency $\omega_{E S R}$, and measuring an electrical current as a function of the applied magnetic field $B$. Thus, the current will show a single peak either at $B_{-}=\hbar\left(\omega_{\mathrm{ESR}}-A / 2\right) /\left(g_{e} \mu_{e}\right)$ when the donor nuclear spin is aligned along the $B$ field, or at $B_{+}=$ $\hbar\left(\omega_{\mathrm{ESR}}+A / 2\right) /\left(g_{e} \mu_{e}\right)$ when the donor nucleus is aligned against the field. It is therefore believed that the detection of electron spin resonance of a single donor impurity using ultra sensitive EDMR methods will allow the readout of its nuclear spin state, or even of ${ }^{29} \mathrm{Si}$ nuclear spins nearby the donor.

However, it is not clear that this is possible. The problem is that the time it takes to detect EDMR of a single donor spin $t_{\text {EDMR }}$ must be shorter than nu- clear spin-flip time $T_{1 n}$, and as we show below the electric current will induce nuclear spin-flips. Moreover, read-out time must be longer than nuclear spin coherence time $T_{2 n}$ for the nuclear spin wave function to collapse into one of the outcome states. Thus, the EDMR read-out scheme will be limited by a contrast of $\exp \left[-\operatorname{Max}\left(T_{2 n}, t_{\mathrm{EDMR}}\right) / T_{1 n}\right]$. The best possible scenario occurs when $t_{\mathrm{EDMR}}<T_{2 n}$, leading to an optimal read-out contrast of $\exp \left(-T_{2 n} / T_{1 n}\right)$.

In this letter, we describe our theory for the interaction of nuclear spin qubits with a "classical current", i.e. a current formed by an incoherent ensemble of conduction electrons, the one used in common electronic devices such as EDMR.

Consider a donor impurity interacting with an electron gas $[3$ 12]. Scattering events preserve the total spin of conduction and localized electrons, leading to the interaction Hamiltonian [17]

$$
\begin{aligned}
\mathcal{H}_{\mathrm{ce}}= & J \sum_{k, k^{\prime}}\left[S_{-} c_{k^{\prime} \uparrow}^{\dagger} c_{k \downarrow}+S_{+} c_{k^{\prime} \downarrow}^{\dagger} c_{k \uparrow}\right. \\
& \left.+S_{z}\left(c_{k^{\prime} \uparrow}^{\dagger} c_{k \uparrow}-c_{k^{\prime} \downarrow}^{\dagger} c_{k \downarrow}\right)\right] .
\end{aligned}
$$

Here $c_{k \sigma}^{\dagger}, c_{k \sigma}$ are creation/annihilation operators for a conduction electron with momentum $k$ and spin $\sigma=\uparrow$ or $\downarrow, S_{ \pm}=S_{x} \pm i S_{y}$ are spin raising/lowering operators for the donor electron, and $J$ is their "bare" exchange interaction.

Exchange scattering will tend to equilibrate the localized spin with the electron gas. If the localized spin is not in thermal equilibrium, its expectation value $\left\langle S_{\alpha}\right\rangle$ along any direction $\alpha=x, y, z$ will decay exponentially in time according to the rate,

$$
\Gamma_{e} \equiv \frac{1}{T_{1 e}}=\frac{1}{T_{2 e}}=\frac{2 \pi}{\hbar}\left|J_{\mathrm{eff}} \nu\right|^{2} \hbar \omega_{e} \operatorname{coth}\left(\frac{\hbar \omega_{e}}{2 k_{B} T}\right)
$$

where $\nu$ is the energy density of states at the Fermi level. The effective exchange interaction $J_{\text {eff }}$ is obtained after summing and renormalizing all orders of perturbation theory in $J \nu$, resulting in an universal expression that 


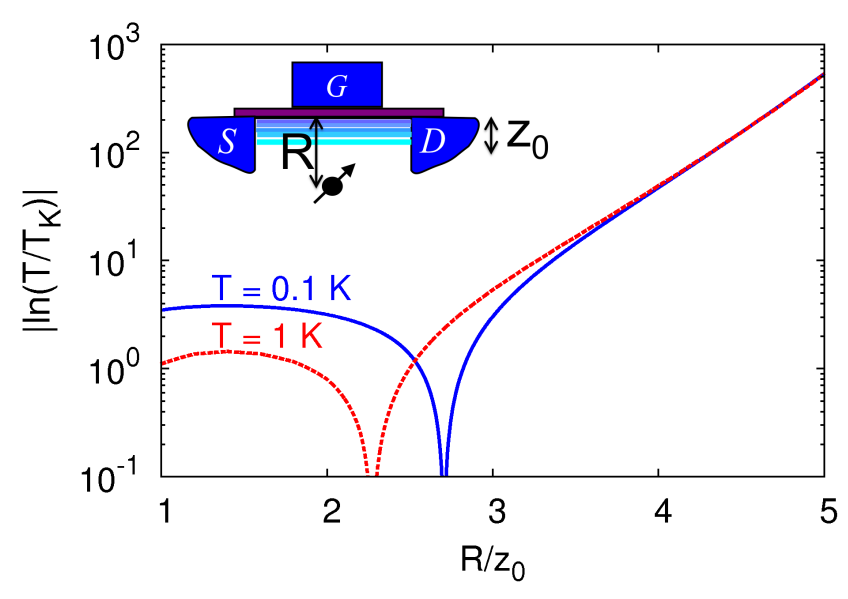

FIG. 1. (color online) This figure demonstrates how a donor impurity implanted in a silicon accumulation field effect transistor (inset) can be tuned into and out of the Kondo regime using a top gate voltage. The plot shows $\left|\ln \left(T / T_{K}\right)\right|$ for $T=0.1 \mathrm{~K}$ and $T=1 \mathrm{~K}$ for a single donor impurity fixed at a distance $R$ from the transistor interface (model assumptions and parameters are the same as Fig. 7 of [] $)$. The length scale $z_{0}$ models the two-dimensional electron gas width, that is controllable electrically over the range $z_{0} \approx 30-100 \AA$. Hence a donor can be tuned from $R / z_{0} \approx 3$ (strong coupling regime) to $R / z_{0} \approx 5$ (weak coupling regime) by increasing the top gate voltage. Recently, a similar electrical tuning of the donor Kondo temperature was demonstrated in a side-gated silicon device [20].

depends only on the Kondo temperature $T_{K}$ [17, 18]:

$$
\left|J_{\mathrm{eff}} \nu\right|^{2}=\left[\pi^{2}+\frac{4}{3}\left|\ln \left(\frac{T}{T_{K}}\right)\right|^{2}\right]^{-1} .
$$

Calculations of the Kondo temperature for realistic parameters of a silicon transistor are presented in Figure 1 .

Equation (3) is valid for $T>T_{K}$. When $T<T_{K}$, the conduction electron spins will screen out the donor electron spin, forming a Kondo singlet. This will add non-exponential decay to $\left\langle S_{\alpha}\right\rangle$, with a rate $\Gamma_{e} \sim k_{B} T_{K} / \hbar$ [19].

At $T>T_{K},\left\langle S_{\alpha}\right\rangle$ decays exponentially in time, towards its thermal equilibrium value. This leads to the following effective model for donor electron plus nuclear spin evolution subject to the conduction electron environment [21]:

$$
\frac{\partial \hat{\rho}}{\partial t}=-i\left[\mathcal{H}_{e n}, \hat{\rho}\right]+\Gamma_{e}\left(\sum_{\alpha=x, y, z} S_{\alpha} \hat{\rho} S_{\alpha}-\frac{3}{4} \hat{\rho}+p_{e} S_{z}\right) .
$$

Here $\hat{\rho}$ is the density matrix describing donor electron plus nuclear spin, $\Gamma_{e}$ is given by Eq. (3), and $p_{e}=$ $-\tanh \left[\hbar \omega_{e} /\left(2 k_{B} T\right)\right]$ is the equilibrium donor electron spin polarization. With the usual definitions $\langle\boldsymbol{S}\rangle=$
$\operatorname{Tr}\{\hat{\rho} \boldsymbol{S}\},\langle\boldsymbol{I}\rangle=\operatorname{Tr}\{\hat{\rho} \boldsymbol{I}\}$, and $\langle\boldsymbol{S} \boldsymbol{I}\rangle=\operatorname{Tr}(\hat{\rho} \boldsymbol{S} \boldsymbol{I})$ (a matrix formed by the outer product between $\boldsymbol{S}$ and $\boldsymbol{I}$ ) we can put Eq. (5) in a more convenient form that we call the generalized Bloch equation for the donor:

$$
\begin{aligned}
\langle\dot{\boldsymbol{S}}\rangle= & \omega_{e} \hat{\boldsymbol{z}} \times\langle\boldsymbol{S}\rangle-A\langle\boldsymbol{S} \times \boldsymbol{I}\rangle-\Gamma_{e}\left(\langle\boldsymbol{S}\rangle-\frac{p_{e}}{2} \hat{\boldsymbol{z}}\right) \\
\langle\dot{\boldsymbol{I}}\rangle= & -\omega_{n} \hat{\boldsymbol{z}} \times\langle\boldsymbol{I}\rangle+A\langle\boldsymbol{S} \times \boldsymbol{I}\rangle \\
\langle\dot{\boldsymbol{S}} \boldsymbol{I}\rangle= & \omega_{e} \hat{\boldsymbol{z}} \times\langle\boldsymbol{S} \boldsymbol{I}\rangle+\omega_{n}\langle\boldsymbol{S} \boldsymbol{I}\rangle \times \hat{\boldsymbol{z}} \\
& +\frac{A}{4}(\boldsymbol{S}-\boldsymbol{I}) \cdot \boldsymbol{\varepsilon}-\Gamma_{e}\langle\boldsymbol{S} \boldsymbol{I}\rangle
\end{aligned}
$$

with $\varepsilon=\varepsilon_{\alpha \beta \gamma} \hat{\boldsymbol{e}}_{\alpha} \hat{\boldsymbol{e}}_{\beta} \hat{\boldsymbol{e}}_{\gamma}$ the Levi-Civita tensor.

We can solve Equations (6a)- (6c) analytically by moving to a reference frame where the electron spin rotates with frequency $\omega_{e}$ and the nuclear spin rotates with $-\omega_{n}$; within order $\left(A / \omega_{e}\right)^{2}$ we get

$$
\begin{aligned}
\left|\left\langle\dot{\boldsymbol{I}}_{\perp}^{\prime}\right\rangle\right| & \approx-\frac{1}{2} \frac{\Gamma_{e}}{1+2\left(\frac{\Gamma_{e}}{A}\right)^{2}}\left|\left\langle\boldsymbol{I}_{\perp}^{\prime}\right\rangle\right|, \\
\left\langle\dot{I}_{z}\right\rangle & \approx-\frac{\Gamma_{e}}{1+2\left(\frac{\Gamma_{e}}{A}\right)^{2}+2\left(\frac{\tilde{B}}{A}\right)^{2}}\left(\left\langle I_{z}\right\rangle-\frac{p_{e}}{2}\right),
\end{aligned}
$$

where $\left\langle\boldsymbol{I}^{\prime}\right\rangle$ is the nuclear spin operator in the rotating frame, and $\tilde{B}=\left(\omega_{e}+\omega_{n}\right)$. The decay rate of Eq. (7a) is the new nuclear spin decoherence rate induced by the current,

$$
\frac{1}{T_{2 n}}=\frac{1}{2} \frac{\Gamma_{e}}{1+2\left(\frac{\Gamma_{e}}{A}\right)^{2}} .
$$

In the limit $\Gamma_{e} \ll A$, we get $1 / T_{2 n} \approx \Gamma_{e} / 2$ showing that the nuclear spin "follows" the electron. However, in the opposite regime of $\Gamma_{e} \gg A$ we get a decoherence rate that is inversely proportional to $\Gamma_{e}: 1 / T_{2 n} \approx A^{2} / \Gamma_{e}$, signaling a transition to a motional narrowing regime. Here the electron spin fluctuates so fast that its effect on the nuclear spin's phase is averaged out.

Similarly, the decay rate of Eq. (7b) is the new nuclear spin-flip rate,

$$
\frac{1}{T_{1 n}}=\frac{\Gamma_{e}}{1+2\left(\frac{\Gamma_{e}}{A}\right)^{2}+2\left(\frac{\tilde{B}}{A}\right)^{2}} .
$$

As $\Gamma_{e}$ increases, $1 / T_{1 n}$ peaks at $\Gamma_{e}=\tilde{B} \approx \omega_{e}$. The origin of this effect is a transfer of population from the electron spin to the nucleus assisted by the "flip-flop" coherence $\left\langle S_{-} I_{+}\right\rangle$. It is necessary for the electron spin fluctuation frequency $\Gamma_{e}$ to match the flip-flop coherence $\tilde{B}$ for the transfer to occur. The net result is a transfer of electron spin polarization $p_{e}$ into the nuclear spin.

This result suggests a method for qubit initialization via interaction with the electron gas. The idea is to electrically tune the Kondo temperature so that $\Gamma_{e}$ gets close to $\omega_{e}$. This can be achieved when $\ln \left(T / T_{K}\right) \approx$ $\sqrt{3 \pi /\left(2\left|p_{e}\right|\right)}$ or $T \approx 10 T_{K}$ for $\left|p_{e}\right|=1$, and we call 
this the "strong coupling regime" $\left(\Gamma_{e} \approx \omega_{e}\right)$. Figure 1 describes how to tune into this regime with a top gated device. In the strong coupling regime we get $\operatorname{Max}\left(1 / T_{1 n}\right) \approx A^{2} /\left(4 \omega_{e}\right)$. For a phosphorus donor in silicon, $A=120 \mathrm{MHz}$ and $\omega_{e}=28 \mathrm{GHz}$ for $B=1 \mathrm{~T}$, leading to qubit initialization times $T_{1 n}=1 \mu \mathrm{s}$. These electrical initialization times are several orders of magnitude faster than other methods previously considered for donor nuclear spins in silicon. In [22], white light was used to polarize donor nuclei within $150 \mathrm{~s}$, while [23] used a laser tuned to an exciton transition to initialize within $0.5 \mathrm{~s}$.

We remark that the nuclear spin-flip process described by Eq. (7b) results from an "indirect" interaction of the donor nucleus with conduction electrons, mediated by the donor electron spin through hyperfine coupling $A$. There also exists an additional "direct" interaction between the donor nuclear spin and the conduction electrons: It gives rise to the Korringa relaxation mechanism [24], that is usually quite weak for semiconductors. We calculated the Korringa rate for the phosphorus donor in the silicon transistor shown in Fig. 1 We obtained $\left(1 / T_{1 n}\right)_{\text {Korringa }}<10^{-3} \mathrm{~s}^{-1}$ for $R / z_{0}>2$ at low temperature $(T<10 \mathrm{~K})$. Hence, at times shorter than a few minutes we may neglect this "direct" interaction between the donor nucleus and the electron gas, and the donor nuclear spin polarization will remain close to the electron's, $p_{n} \approx p_{e}=-\tanh \left(\hbar \omega_{e} / 2 k_{B} T\right)$. However, we note that the Korringa rate will eventually drive the nuclear spin back to its thermal equilibrium state, with small polarization $p_{n}=+\tanh \left(\hbar \omega_{n} / 2 k_{B} T\right)$.

We now describe nuclear spin read-out with EDMR. The donor spin is irradiated with a resonant microwave field of frequency $\omega_{\mathrm{ESR}}$ and amplitude $B_{\perp}$, inducing electron spin precession with frequency $\Omega_{\perp}=g_{e} \mu_{e} B_{\perp} / \hbar$. Only electron spins satisfying the equal population or "saturation" condition $\Omega_{\perp} \gtrsim \Gamma_{e}$ can be detected [1], and optimal sensitivity is achieved at the threshold for saturation, $\Omega_{\perp}=\Gamma_{e}$ [8]. In terms of the Kondo temperature, optimal EDMR takes place for $\ln \left(T / T_{K}\right)=$ $\sqrt{\left[3 \pi /\left(2\left|p_{e}\right|\right)\right]\left(B / B_{\perp}\right)}$, a regime that requires low Kondo temperature. We call this limit the "weak coupling" regime. For $B=1 \mathrm{~T}$ and $B_{\perp}=0.3 \mathrm{G}$, weak coupling requires $\ln \left(T / T_{K}\right) \approx 500$.

We generalized Eqs. (6a) -6c for the case of resonant excitation; when $\Omega_{\perp} \gtrsim \Gamma_{e}$ and $\Omega_{\perp} \ll A$, the nuclear spin relaxation rates are given by Eqs. (7a $-(7 \mathrm{~b})$ with $\Gamma_{e} \rightarrow \Omega_{\perp}$. Hence we have $T_{1 n} \approx 2\left(\omega_{e} / A\right)^{2} / \Omega_{\perp}$ and $T_{2 n} \approx 2 / \Omega_{\perp}$. From this result we can deduce the maximum possible contrast for read-out of nuclear spin states: Nuclear spin-flips during read-out will lead to contrast equal to $\exp \left(-T_{2 n} / T_{1 n}\right)=\exp \left[-\left(A / \omega_{e}\right)^{2}\right]$, that is quite close to 1 when $\omega_{e} \gg A$. We recall that this optimum contrast occurs only when $t_{\mathrm{EDMR}}$, the time it takes to detect EDMR of a single donor, is less than $T_{2 n}$.

A more likely scenario is that $t_{\mathrm{EDMR}}$ will be quite long.
Assuming the longest acceptable $t_{\mathrm{EDMR}}$ is equal to $T_{1 n}$, we now derive a sensitivity condition for EDMR detection of nuclear spins. Such a condition will depend critically on the value of the EDMR current amplitude $(\Delta I)_{\mathrm{EDMR}}$, that is given by the current difference when only one donor is on and off resonance with a microwave field. A key observation is that $(\Delta I)_{\text {EDMR }}$ must be larger than the shot noise $(\Delta I)_{\text {shot }}$ accumulated during $T_{1 n}$ :

$$
\left(\frac{\Delta I}{I}\right)_{\mathrm{EDMR}}>\left(\frac{\Delta I}{I}\right)_{\mathrm{shot}}=\frac{1}{\sqrt{N\left(T_{1 n}\right)}},
$$

where $I$ is the average device current and $N\left(T_{1 n}\right)=$ $I T_{1 n} / e$ is the number of electrons that passed through the device during $T_{1 n}$. Using the value of $T_{1 n}$ computed above we get the sensitivity criterion for nuclear spin read-out:

$$
\left(\frac{\Delta I}{I}\right)_{\mathrm{EDMR}}>\sqrt{\frac{e \Omega_{\perp}}{2 I}}\left(\frac{A}{\omega_{e}}\right) .
$$

We note that an implicit assumption of Eq. (11) is that $A>1 / T_{2 e}^{*}$, i.e., the separation of the two EDMR peaks is larger than their linewidth. For $I=1 \mu \mathrm{A}, B_{\perp}=0.3 \mathrm{G}$ and $B=1 \mathrm{~T}$ we get $(\Delta I / I)_{\mathrm{EDMR}}>3 \times 10^{-6}[26]$.

A recent theory of the spin-dependent scattering mechanism of EDMR [8] predicted $(\Delta I / I)_{\mathrm{EDMR}}=6 \times 10^{-6} \times$ $\tanh \left(\hbar \omega_{e} / 2 k_{B} T\right) \times(B / 1 \mathrm{~T}) \times\left(\mathrm{B}_{\perp} / 0.3 \mathrm{G}\right)$, for a single donor implanted in a transistor of area $0.1(\mu \mathrm{m})^{2}$. At $B \sim 1 \mathrm{~T}$ and $T=1 \mathrm{~K}$ this is right at the sensitivity limit; however, much better sensitivity can be obtained by going to higher $B$ fields.

There exists other methods for EDMR detection that can potentially yield much higher $(\Delta I / I)_{\mathrm{EDMR}}$, most notably the ones based on optical excitation and recombination [1, 33 5, 7]. Electron-hole pairs are excited by light and the photocurrent is monitored as a function of magnetic field. In this case additional relaxation channels such as capture of an extra donor electron with rate $\Gamma_{c}$, with corresponding ionization of the extra electron with rate $\Gamma_{i}$ can be present. It is straightforward to generalize our theory to these cases; we obtain the same expressions as above with the substitution $\Gamma_{e} \rightarrow\left(\Gamma_{e}+\Gamma_{i}+\Gamma_{c}\right)$ and $A^{2} \rightarrow\left\langle A^{2}\right\rangle=A^{2} \Gamma_{i} /\left(\Gamma_{i}+\Gamma_{c}\right)$ (effective hyperfine coupling gets reduced by capture, because a singlet produces zero hyperfine field on the nuclear spin). With these substitutions, Eqs. (8) - (11) can be applied to recombination based EDMR.

In a typical transistor, the gate voltage can be used to control the overlap between the electron gas and the donor impurity wavefunction. Thus, the Kondo temperature can be controlled electrically from $T_{K}=0$ (when the electron gas density is zero, i.e., the transistor is turned off) all the way to large $T_{K}$. This is demonstrated for a top gated device 12, 25] in Figure 1. Hence, we argue that it is possible to switch donors electrically into and out of three distinct modes: In the "quantum evolution" 
mode, donors have no overlap with the electron gas, implying $T_{K}=0$ and $T_{1 n}, T_{2 n}$ are longer than a few seconds [15]. For larger gate voltage, overlap is small and the donor is in the weak coupling regime, allowing quantum read-out. For even larger gate voltages, the donor will be in the strong coupling regime enabling fast initialization.

In conclusion, we presented a theory for the interaction of an electric current with donor nuclear spin qubits. We showed that electric tuning of donors away from the Kondo regime allows coherent evolution and high contrast quantum read-out using electrically detected magnetic resonance; in contrast, electric tuning close to the Kondo regime enables fast initialization of nuclear spin qubits. We derived a general sensitivity criterion for the read-out of nuclear spin states using EDMR.

We thank C.C. Lo, J.J.L. Morton, T. Schenkel, T. Tiedje, and L.H. Willems van Beveren for useful suggestions to this work. Our research was supported by the NSERC Discovery program.

* rdesousa@uvic.ca

[1] A. Honig, Phys. Rev. Lett.17, 186 (1966); J. Schmidt and I. Solomon, C.R. Acad. Sci. B 263, 169 (1966); D. Kaplan, I. Solomon, and N.F. Mott, J. Phys. (Paris), Lett. 39, L51 (1978).

[2] B.E. Kane, Nature 393, 133 (1998).

[3] C. Boehme and K. Lips, Phys. Rev. Lett.91, 246603 (2003).

[4] D.R. McCamey, H. Huebl, M.S. Brandt, W.D. Hutchison, J.C. McCallum, R.G. Clark, and A.R. Hamilton, Appl. Phys. Lett.89, 182115 (2006).

[5] A.R. Stegner, C. Boehme, H. Huebl, M. Stutzmann, K. Lips, and M.S. Brandt, Nature Physics 2, 835 (2007).

[6] M. Sarovar, K.C. Young, T. Schenkel, and K.B. Whaley, Phys. Rev. B78, 245302 (2008).

[7] G.W. Morley, D.R. McCamey, H.A. Seipel, L.-C. Brunel, J. van Tol, and C. Boehme, Phys. Rev. Lett.101, 207602 (2008).

[8] R. de Sousa, C.C. Lo, J. Bokor, Phys. Rev. B80, 045320 (2009).

[9] H. Morishita, L.S. Vlasenko, H. Tanaka, K. Semba, K. Sawano, Y. Shiraki, M. Eto, and K.M. Itoh, Phys. Rev.
B80, 205206 (2009).

[10] D.R. McCamey, J. Van Tol, G.W. Morley, and C. Boehme, Science 330, 1652 (2010).

[11] F. Hoehne, L. Dreher,H. Huebl, M. Stutzmann, and M.S. Brandt, Phys. Rev. Lett.106, 187601 (2011).

[12] C. C. Lo, V. Lang, R.E. George, J.J.L. Morton, A.M. Tyryshkin, S.A. Lyon, J. Bokor, and T. Schenkel, Phys. Rev. Lett.106, 207601 (2011).

[13] F. Jelezko, T. Gaebel, I. Popa, M. Domhan, A. Gruber, and J. Wrachtrup, Phys. Rev. Lett.93, 130501 (2004); L. Childress, M. V. Gurudev Dutt, J. M. Taylor, A. S. Zibrov, F. Jelezko, J. Wrachtrup, P. R. Hemmer, and M. D. Lukin, Science 314, 281 (2006).

[14] T.D. Ladd, D. Maryenko, and Y. Yamamoto, E. Abe, and K. M. Itoh, Phys. Rev. B71, 014401 (2005).

[15] J.J.L. Morton, A.M. Tyryshkin, R.M. Brown, S. Shankar, B.W. Lovet, A. Ardavan, T. Schenkel, E.E. Haller, J.W. Ager, and S.A. Lyon, Nature 455, 1085 (2008).

[16] A. Morello et al., Nature 467, 687 (2010).

[17] A. C. Hewson, The Kondo Problem to Heavy Fermions (Cambridge University Press, Cambridge, England, 1993).

[18] C. Van Haesendonck, J. Vranken, and Y. Bruynseraede, Phys. Rev. Lett.58, 1968 (1987).

[19] D. Lobaskin and S. Kehrein, Phys. Rev. B71, 193303 (2005); F.B. Anders and A. Schiller, Phys. Rev. B74, 245113 (2006).

[20] G.P. Lansbergen, G.C. Tettamanzi, J. Verduijn, N. Collaert, S. Biesemans, M. Blaauboer, and S. Rogge, Nano Lett. 10, 455 (2010).

[21] This model can be formally derived within the BornMarkov approximation (second order in $J$ ), see Y. Rikitake and H. Imamura, Phys. Rev. B72, 033308 (2005).

[22] D.R. McCamey, J. van Tol, G.W. Morley, and C. Boehme, Phys. Rev. Lett.102, 027601 (2009).

[23] A. Yang et al., Phys. Rev. Lett.102, 257401 (2009).

[24] See section IX-I-A of A. Abragam, Principles of Nuclear Magnetism (Oxford University Press, Oxford, England, 1961).

[25] C.C. Lo, J. Bokor, T. Schenkel, J. He, A.M. Tyryshkin, and S.A. Lyon, Appl. Phys. Lett.91, 242106 (2007).

[26] We do not expect "excess" noise (due to other sources, e.g. $1 / f$ noise) to be larger than shot noise during readout. In [25], EDMR was measured in a high quality transistor using lock-in detection at $1 \mathrm{KHz}$; the excess noise after averaging for several minutes was $\Delta I / I \approx 10^{-8}$ (see Fig. 2c in [25]). We remark that this is much lower than our shot noise estimate $(\Delta I / I)_{\text {shot }}=3 \times 10^{-6}$. 